\title{
Nouvelles formes d'occupation de l'espace, la place de la Chine et de l'Inde
}

Isabelle Sourbès Verger ${ }^{1}$

Résumé: Pour la Chine comme pour l'Inde, les activités spatiales tiennent une place importante dans leur politique nationale de développement depuis les années 1980-1990. Les avantages des technologies spatiales sont en effet essentiels pour ces deux Etats de vaste superficie, très peuplés, manquant d'infrastructures terrestres et soucieux de développer leurs capacités pour une meilleure mise en valeur de leur territoire et de leurs ressources. A côté de ces préoccupations économiques communes, la Chine et l'Inde privilégient des politiques spatiales différentes liées à leurs caractéristiques politiques et socio-économiques propres induisant entre autres une relation très différente avec les autres puissances spatiales. En 2019, la Chine et l'Inde se différencient donc par une organisation interne et des priorités affichées profondément différentes. Pour autant, la maturité croissante de leur secteur spatial laisse apparaitre des tendances proches, dans le domaine de l'exploration ou des vols habités. En revanche, l'intérêt accordé par la Chine au développement d'activités spatiales militaires et plus largement au spatial comme symbole de puissance reste une rupture fondamentale par rapport à la politique indienne qui évolue vers un effort accru de commercialisation et de développement de capacités industrielles au-delà de l'agence spatiale, l'ISRO, jusque-là au cour de tout le dispositif.

1 Centre Alexandre Koyré - CNRS-EHESS-MNHN - Paris - França - isabelle.sourbes@cnrs.fr 
Mots-lés: Exploration spatial, Politique spatiale chinoise, Oolitique spatiale indienne.

\section{New forms of space occupation, the place of China and India}

Abstract: For both China and India, space activities have been an important part of their national development policy since the 1980 s and 1990s. The advantages of space technologies are indeed essential for these two populated and vast States, whose lack of terrestrial infrastructures make them anxious to develop capacities for a better enhancement of their territory and their resources. Sharing common economic concerns, China and India promote different spatial policies related to their own political and socio-economic characteristics, inducing, among other things, a very different relationship with other space powers. In 2019, China and India are thus differentiated by an internal organization and deeply different priorities. However, the maturity of their space sector reveals similar trends, both in the fields of exploration or manned flights. On the other hand, China's interest in the development of military space activities and, more broadly, to use space as a symbol of power, remains a fundamental rupture from Indian policy, which evolves towards the commercialization and development of industrial capabilities beyond the ISRO space agency, the heart of its whole spacial policy.

Keywords: Space exploration, Chinese space policy, Indian space policy

\section{Novas formas de ocupação do espaço, o lugar da China e da Índia}

Resumo: Tanto para a China como para a Índia, as atividades espaciais adquiriram destacada posição em suas políticas nacionais de desenvolvimento após os anos 1980-1990. Os benefícios das tecnologias espaciais são essenciais para esses dois Estados de vasta superfície, muito populosos, sem infraestrutura terrestre e preocupados com o desenvolvimento de suas capacidades para o melhor aproveitamento de seu território e recursos naturais. Ao lado de suas comuns preocupações econômicas, China e Índia privilegiam diferentes políticas espaciais ligadas às suas características políticas e socioeconômicas. Em 2019, com a maturidade de seus programas espaciais, China e Índia revelam tendências semelhantes no domínio da exploração e dos voos tripulados, embora possuam diferentes organizações internas e prioridades. O interesse chinês no desenvolvimento de atividades espaciais militares e, mais amplamente, no espaço como 
um símbolo de poder, marca uma ruptura fundamental em relação à política indiana, que evolui no sentido de ampliar seu caráter comercial e desenvolver suas capacidades industriais para além de sua agência espacial, até então no centro de toda sua política espacial.

Palavras-chave: Exploração espacial, Política espacial chinesa, Política espacial indiana.

Lorsque L'Union des républiques socialistes soviétiques (URSS) lance le premier satellite Spoutnik, le 4 octobre 1957, l'objectif est d'abord scientifique puisqu'il s'agit, dans le cadre de l'Année Géophysique Internationale ${ }^{2}$, de contribuer à létude des propriétés physiques de la haute atmosphère et de compléter ainsi les observations réalisées sur terre par d'autres moyens comme les stations polaires, bateaux ... (Coulomb, 1956).

A cette époque, seuls les États-Unis et l'URSS essayent alors de maîtriser les moyens d'accès à l'espace et tous les deux annoncent qu'ils vont lancer un satellite. L'Union soviétique na rejoint le Conseil scientifique des Unions internationales ${ }^{3}$ qu'après la mort de Staline mais la Chine n'en est pas membre pour protester contre la reconnaissance de Taïwan. L'Inde est, elle, représentée dans ce réseau prestigieux de la science internationale par un scientifique connu, Vikram A. Sarabhai ${ }^{4}$, spécialiste de la physique solaire et du rayonnement cosmique formé à Cambridge au Royaume-Uni.

Être capable draccéder à lespace confère alors un statut exceptionnel sur la scène internationale. En ouvrant avec Spoutnik la voie à lexploration de l`espace extra-atmosphérique, l`Union soviétique remettait symboliquement en cause la supériorité technologique des États-Unis dans un domaine dont il était clair pour tous quil allait, avec le nucléaire ${ }^{5}$, déterminer le statut de super-

2 L'Année Géophysique Internationale, elle est aussi connue comme le Troisième Année Polaire Internationale.

3 Le Conseil International des Unions Scientifiques (ICSU, en anglais) fut créé à Bruxelles en 1931, pour promouvoir l'activité scientifique internationale dans les différentes branches des sciences et techniques et son application dans l'intérêt de l'humanité.

4 Membre d'une influente famille indienne de riches industriels progressistes, l'influence et l'énergie de Vikram A. Sarabhai sont citées par tous les témoins comme phénoménales et vont catalyser le développement de la science spatiale indienne. Il est précurseur du programme national de lanceur et de satellites nationaux développé dans les années 1970-1980.

5 Cette relation très étroite alors entre le nucléaire et l'espace tenait à plusieurs facteurs: la proximité technologique entre un lanceur et un missile intercontinental (ICBM) capable de délivrer une bombe nucléaire, le potentiel d’observation à distance sans autorisation préalable du territoire de l'adversaire pour le cartographier et préparer d'éventuels ciblages... D’un autre côté, la mise en place de systèmes spatiaux 
puissance. En multipliant les Premières pendant la première décennie (sonde atteignant la Lune, photos de la face cachée de la Lune, premiers homme et femme à l'espace, première sortie d'un cosmonaute dans l'espace hors de son vaisseau...), l'URSS insiste sur l'adéquation entre son modèle marxiste scientifique et la transposition concrète dans l'espace du concept «d'homme nouveau». Cet usage idéologique est particulièrement problématique pour les Occidentaux dans le contexte sensible de la décolonisation et de la concurrence des modèles qui sont alors proposés aux nouveaux États.

Cette dimension directement géopolitique de l'espace va perdurer jusquau premier pas triomphal d'un Américain sur la Lune, Neil Armstrong, présenté à son tour, en juillet 1969, 17 ans après Gagarine, comme le représentant de toute l'humanité. Le programme Apollo sanctionne désormais le statut de première puissance spatiale des États-Unis, un statut exceptionnel toujours actuel.

La perception des activités spatiales en 2019 est bien différente. Si les enjeux de prestige nont pas disparu, comme le montre le traitement médiatique de quelques missions d'exploration, les usages de l'espace se sont désormais banalisés. L'existence de satellites orbitant autour de la Terre ne suscite aucun intérêt particulier. Il faut la démesure de projets de constellations de satellites de plusieurs milliers de satellites pour relancer l'attention des médias et du public ou les ambitions affichées des nouveaux acteurs du New Space issus du monde des GAFA $^{6}$ comme Elon Musk ${ }^{7}$ et son projet de colonisation martienne ou Richard Branson $^{8}$ et ses projets de tourisme spatial.

Plus de 60 ans après le début de lère spatiale, le nombre d'États membres actifs du club spatial reste limité. Russie, États-Unis, Japon, Chine, Europe spatiale, Inde, Israël, Iran, Corée du nord et Corée du sud ${ }^{9}$ sont les seuls à maîtriser les moyens d'accès à l'espace et à disposer des moyens industriels pour construire satellites et lanceurs sur une base nationale. En parallèle, les évolutions technologiques, dont la miniaturisation, abaissent le coût de l'accès aux technologies spatiales. Cest particulièrement vrai pour les petits satellites mais pourraient aussi permettre le développement de petits lanceurs. La nature de la puissance spatiale évolue d’autant plus que des sociétés privées annoncent leurs propres

de détection de tirs d'ICBM deviendra un facteur de stabilisation face à la menace de la destruction mutuelle assurée et l'observation par satellite permettra la vérification et donc la signature des accords de limitation des armements SALT/ABM de 1972 et 1978.

6 On appelle GAFA (Google Amazon Facebook Apple) les géants de l'Internet.

7 Ellon Musk, il est le président-directeur général (PDG) de l’enterprise Space-X.

8 Richard Bran, il est PDG de l'enterprise Virgin Galactic.

9 Lordre ici fourni est chronologique mais ne correspond pas à leurs capacités respectives. 
ambitions, indépendamment des programmes nationaux suggérant de nouvelles relations avec les acteurs étatiques au moins pour l'écosystème américain.

Dans ce contexte en évolution, comment des puissances spatiales comme la Chine et l'Inde se positionnent-elles?

\section{La place historique de la Chine et de l'Inde en tant qu'acteurs de la mise en valeur de l'espace}

\subsection{De "nouvelles puissances spatiales" dans les années 1980-1990}

De 1957 à 1970, l'Union soviétique et les États-Unis sont les seuls à maîtriser les technologies spatiales ${ }^{10}$. La mise en valeur de l'espace est profondément marquée par le contexte historique de la guerre froide. Chacun des deux États s’applique alors à expérimenter les différents usages des satellites à des fins prioritairement de sécurité nationale: découverte et analyse des caractéristiques propres du milieu spatial par les satellites scientifiques et technologiques, cartographie et observation du territoire de l'adversaire et de ses ressources par les satellites dits de reconnaissance essentiellement photographiques, amélioration des communications et du transport de l'information par les satellites de télécommunications, surveillance des essais nucléaires et des tirs de missiles.

Les enjeux de politique étrangère représentent une deuxième dimension considérée comme d'autant plus importante que la décolonisation accuse l'importance pour chacun des leaders, du bloc capitaliste comme du bloc communiste, de se poser en modèle.

Cette phase d'apprentissage dans tous les domaines se traduit par l'utilisation d'un nombre varié d’orbites, de celles en orbite basse des satellites de reconnaissance aux premières mise à poste sur l'orbite géostationnaire et aux trajectoires vers la Lune, Mars, Vénus. Elle est aussi marquée par une présence de plus en plus grande des activités civiles avec la montée en puissance de la $\mathrm{NASA}^{11}$ destinée à contribuer à l'affichage du leadership des États-Unis alors que du côté soviétique l'ensemble des activités relève du complexe militaro-industriel avec une implication particulière de l'Académie des Sciences.

10 La France accomplit en 1965 la mise sur orbite du satellite Astérix avec un lanceur Diamant depuis Hammaguir devenant ainsi la zème puissance spatiale mais ne poursuit pas au niveau national et choisit la voie de la coopération au travers de sa participation à l'Agence spatiale européenne.

11 National Aeronautics and Space Administration (NASA) c'est l'agence gouvernementale civile créée en 1958 pour conduire le programme spatial américain. 
Ce duopole se clôt dans les années 1970 par une première ouverture du club spatial à des acteurs asiatiques avec les premiers tirs nationaux du Japon ${ }^{12}$ puis, 10 semaines plus tard, de la Chine ${ }^{13}$. Cette démonstration vise à la reconnaissance comme grande puissance concrétisant l'ambition énoncée par Mao dès 1956, avant même le lancement du 1er satellite soviétique. La Chine affiche ainsi sa volonté dêtre présente dans les deux domaines technologiques les plus prestigieux du XXIe siècle, le nucléaire et l'espace, la maitrise du lancement spatial témoignant aussi de ses capacités dans le domaine des missiles. Pour autant, les bouleversements de la Révolution culturelle pénalisent les projets spatiaux, la rivalité politique entre les factions se traduisant par la mise en place de deux filières concurrentes de lanceurs ${ }^{14}$, l’une à Pékin, l'autre à Shanghai alors que les ressources scientifiques et industrielles sont encore extrêmement limitées.

L'Inde, dont l'organisation spatiale se met en place tout au long des années 1970, s'inscrit dans une tout autre logique que celle de la Chine retardant son entrée dans le club spatial d'une dizaine d'années. Alors que la Chine est complètement isolée sur la scène internationale et que, se vivant menacée, elle met la priorité sur son programme de lanceur issu des compétences acquises dans le domaine des missiles, l'Inde met en pratique sa doctrine du non-alignement. Utilisant les possibilités de coopération internationale du fait du choix d'un programme spatial exclusivement civil, elle entreprend de développer d'abord des capacités satellitaires dans le domaine des télécommunications, de la météorologie et de lobservation de la Terre destinées à lui permettre de lutter contre le sous-développement, la priorité du gouvernement Nehru.

Le lancement du SLV- $3^{15}$ n'intervient qu'en 1980 . Il est la toute première étape de l'acquisition par l'Inde de son statut de puissance spatiale pleine et entière. C’est aussi l'époque du premier lancement réussi de la fusée européenne Ariane$1^{16}$. Si l'on assiste bien à une deuxième vague d’ouverture du club spatial, les deux tirs européens et indiens ne sont pas comparables. Ariane-1 est, en effet, conçu pour effectuer des lancements commerciaux de satellites sur l'orbite géostationnaire, une technologie dont l'Inde ne disposera qu'en 2001.

12 Le lancement le 11 février 1970 du satellite Ohsumi (12 kg) par une fusée Lambda développée par l'Institute of Space and Astronautical Science (ISAS).

13 Le lancement le 24 avril 1970 du satellite Dong Fang Hong de $173 \mathrm{~kg}$ par un lanceur Chang Zheng ou Longue Marche (CZ-1).

14 Chang Zhen (Longue Marche) pour Pékin et Feng Bar (Tempête) pour Shanghai.

15 Le lanceur SLV-3, met sur orbite le satellite Rohini de $35 \mathrm{~kg}$ le 18 juillet 1980.

16 Le lanceur Ariane 1 effectue son 1er tir le 24 décembre 1979, il est destiné à placer des satellites de plus d'itonne sur orbite géostationnaire (à $36000 \mathrm{~km}$ de la Terre dans le plan de l’équateur). 
Pendant les années 1980-1990, alors que l'activité soviétique sétiole, que les États-Unis creusent l'écart en termes de capacités du fait d'un investissement public au moins 2 fois supérieur à celui de toutes les autres puissances spatiales réunies $^{17}$, que le spatial occidental propose de plus en plus d'applications civiles sur la scène internationale, la Chine et l'Inde poursuivent chacune leur voie propre avec des ambitions sur le moyen terme.

En Chine, l’arrivée au pouvoir de Deng Xiaoping à la fin de la Révolution culturelle marque un souci de rationalisation des activités, avec un intérêt accru pour le développement des applications satellitaires et la valorisation des compétences déjà acquises. La Chine propose ainsi, en 1985, ses lanceurs sur le marché commercial, une initiative peu couronnée de succès pour des raisons diverses ${ }^{18}$ mais qui répond à une volonté de normalisation sur la scène internationale à des fins d'image mais aussi d'ouverture au monde extérieur. C'est ainsi que la Chine développe un partenariat avec le Brésil dans le cadre de sa politique de coopération Sud-Sud en 1988. Le premier satellite CBERS (China Brazil Earth Resources Satellite) prévu pour 1993 n’a toutefois été lancé qu’en 1999, la mise en œuvre financière et technique du programme savérant plus compliquée que prévu.

Les capacités spatiales de la Chine se développent ainsi progressivement avec la mise au point d'une gamme de lanceurs, la maitrise des satellites géostationnaires, une performance croissante dans le domaine des télécommunications, de l'observation de la Terre, de la météorologie et la récupération de capsules automatiques ainsi que la mise en œuvre d'un programme d'envois d'hommes (et femmes) dans l'espace ${ }^{19}$.

Sur le plan de lorganisation, les orientations générales sont de la responsabilité de la Commission militaire centrale, la gestion du secteur étant sous la tutelle de la Commission scientifique et technique pour la défense nationale (COSTIND, selon le sigle anglais). L'Armée Populaire de Libération est en charge des bases de lancement et des tirs. La commercialisation des tirs est assurée par la Compagnie de la Grande Muraille (CGWIC, selon le sigle anglais) qui émane des deux grandes entreprises d'état du secteur.

17 Les Etats-Unis ont un budget de 40 milliards de dollars, l'Europe de 8 milliards, la Chine de 6 milliards, la Russie de 5 milliards, le Japon de 3 milliards, l'Inde de 1,8 milliards en 2018.

18 Il faut l'autorisation du gouvernement américain pour tout satellite utilisant des composants américains ce qui complique les procédures et par ailleurs la fiabilité des lanceurs chinois nest pas garantie.

19 Le projet 921 Shenzhou profite de la vente par la Russie de ses technologies dans le domaine des vols habités, la fin de l'URSS conduisant les entreprises russes à compenser la fin de leur financement étatique. 
L'Inde se caractérise par une remarquable stabilité de ses institutions, avec la place centrale de son agence spatiale (l'ISRO) dans le dispositif. Celle-ci est, en effet, en charge de la recherche et du développement des technologies spatiales. Elle est aussi impliquée dans le processus de décision, son président étant secrétaire du Département de l'espace directement rattaché aux services du Premier Ministre. Sur le plan industriel, le modèle indien est profondément original puisque l'Agence identifie elle-même les technologies disponibles dans le tissu industriel qu'elle les adapte pour répondre aux contraintes physiques particulières du milieu spatial et quelle met ensuite à disposition les brevets quelle a acquis via une filiale créée spécialement pour les aspects de commercialisation, l’Antrix Corporation Limited.

Laugmentation progressive du budget spatial qui reste cependant modeste ${ }^{20}$ et le consensus politique sur l'intérêt des technologies spatiales pour le développement du pays conduit au développement de nouveaux programmes dans le domaine des satellites comme des lanceurs avec pour objectif une autonomie croissante. L'Inde diversifie ses coopérations mais doit aussi en passer par les exigences de ses partenaires. C'est ainsi que la dénonciation par les États-Unis du contrat passé avec la Russie pour l'acquisition de technologies et licences destinées à la mise au point d'un moteur cryogénique indien pour le lanceur GSLV destiné aux lancements des satellites géostationnaires nationaux INSAT renforcera la volonté d’accéder à une totale indépendance.

A la différence de la Chine, l'Inde ne cherche pas encore à développer la gamme complète des systèmes spatiaux mais concentre ses efforts sur les satellites d'application et leurs lanceurs, avec une attention particulière sur les services aval largement utilisés par les différents ministères et progressivement les particuliers. Elle effectue un nombre limité de lancements mais dispose de satellites performants construits sur place grâce à des transferts de technologie très diversifiés.

\subsection{Des acteurs de plus en plus présents depuis les années 2000}

Si l'on regarde le nombre de lancements de satellites par nationalités, les années 2000 témoignent de l'arrivée de nouveaux acteurs, en particulier asiatiques, et confirment la présence croissante de la Chine et de l'Inde. Après l'effacement russe comparé à la présence soviétique pendant la décennie précédente,

20 Il passe de 100 à 400 millions de dollars pendant la période. 
la poursuite des programmes chinois et, dans une moindre mesure, indiens amorce une remontée progressive du nombre de tirs.

Devenue en 2003 le zème État capable d'envoyer des hommes dans l'espace, la Chine acquiert dès lors une stature internationale qu'elle ne cesse de renforcer. En fait, elle a déjà rendu public en 2000 son premier document officiel de politique spatiale (White paper on Space Policy) dans lequel elle décline ses réalisations passées et annonce ses programmes actuels et futurs. La création d'une agence spatiale chinoise, la Chinese National Space Administration (CNSA) correspond aussi à un souci de normalisation de son organisation. Il ne sagit cependant que d'une entité administrative de taille réduite chargée de signer les contrats avec les partenaires étrangers et de gérer les programmes. La réalité des compétences reste au sein des grandes entreprises qui sont aussi réorganisées afin dêtre plus souples et efficaces et prendre davantage en compte une problématique de rentabilité tout en restant sous contrôle de l'État.

La succession des différents documents de politique spatiale en 2006, 2011 et 2016 en conformité avec les plans quinquennaux présente la même structure avec un rappel des avancées réalisées et une annonce des programmes en cours. Au final, c'est plus de 20 ans de politique spatiale qui sont ainsi programmées montrant la remarquable continuité de sa politique de catch up en diversifiant de plus en plus ses programmes afin de maitriser la totalité des compétences spatiales. C'est ainsi qu'en parallèle du développement d'une gamme complète de lanceurs et une diversification des bases de lancement, elle développe un système de navigation/localisation type GPS, Beidou, et multiplie les satellites d’observation de la Terre (dotés de capteurs optiques et radar) et de télécommunications destinés à remplacer l'infrastructure terrestre dont elle ne dispose pas. Elle poursuit par ailleurs un programme d'occupation humaine de l'espace avec la construction d'une station spatiale en orbite pour et envisage une exploration automatique à partir de 2017 puis, éventuellement, habitée de la Lune.

Longtemps considérées comme stratégiques et intégrées dans le complexe militaro-commercialo-industriel, les activités spatiales se normalisent progressivement. C'est ainsi qu'une nouvelle base est construite à Wenchang dans la presqu'île de Hainan pour remplacer - en particulier pour les lancements de satellites géostationnaires compte tenu de sa latitude - la base de Xichang, condamnée à la fermeture qui avait été choisie dans la profondeur du territoire pour en assurer la sécurité en cas de conflit. ${ }^{21}$

21 Les premiers étages de lanceur tirés de la base de Xichang retombant sur des zones habitées. 
Sur le plan extérieur, depuis le rapport $\operatorname{Cox}^{22}$ de 1998, la Chine est à nouveau confrontée au refus américain de tout transfert de technologie. C'est donc en mode autonome quelle développe ses propres technologies ce qui présente l'avantage de lui laisser toute liberté quant aux usages qu'elle peut en faire visà-vis de pays tiers. Cela lui permet de jouer un rôle important dans le cadre de lorganisation régionale Asia-Pacific Space Organisation (APSCO) dont elle est leader.

Sur le plan commercial, elle favorise les premiers achats de satellites chinois par des États interdits d’accès à des produits spatiaux par les États-Unis comme le Nigéria, le Nicaragua ou le Venezuela. La compétence spatiale chinoise devient alors un élément d'influence et s'intègre dans des échanges plus larges comme la fourniture dénergie ou de matières premières. Mieux encore, ce positionnement renforce l'image que la Chine cherche à promouvoir d'un acteur indépendant ne prétendant pas intervenir dans les affaires nationales de son partenaire.

Le décalage initial demeurant, c'est à partir des années 2010, que l'Inde développe de nouvelles ambitions dans le domaine de l'exploration et des vols habités. Celles-ci sont présentées par l'agence spatiale indienne (l'ISRO) comme un nouveau type de réalisations venant, non plus en soutien direct de la mise en valeur du territoire, mais comme une volonté de contribution à la reconnaissance du pays en tant que nouvelle puissance globale à la hauteur des pays développés.

Les soucis d'économie restent toutefois bien présents. En témoigne le trajet de la sonde Magalyaan, lancée en novembre 2013 à destination de Mars. Sa satellisation réussie autour de la planète rouge en 2014, après une succession de changements de trajectoires pour compenser les limites de puissance du Polar Satellite Launch Vehicle (PSLV) destiné à des tirs en orbite basse, a donné au spatial indien une nouvelle visibilité internationale. C'était d'ailleurs un des objectifs de ce programme décidé aussi dans l'optique de concurrencer la nouvelle notoriété de la Chine comme acteur spatial de premier plan. Il lui a aussi permis de mettre en avant l'originalité d'une approche "d'innovation frugale" (jugaad) ${ }^{23}$.

Sur le plan de l'autonomie, la réussite en 2014 du lanceur de satellites géostationnaires (Geosynchronous Satellite Launch Vehicle, GSLV), après plus de 20

22 Ce rapport qui porte le nom de son auteur et reste controversé visait à dénoncer l'espionnage mené par la Chine dans le domaine des technologies nucléaires en particulier.

23 La sonde a coûté 55 millions d'euros, chiffre à comparer à celui du rover martien américain Curiosity de près de 2,5milliards de dollars. 
ans d'efforts, marque une étape importante. L'Inde peut désormais assurer seule ses lancements sur l'orbite géostationnaire, même s'il lui faut encore augmenter les capacités du GSLV pour correspondre à la masse de ses derniers satellites INSAT.

L'Inde comme la Chine, même si c'est dans une moindre mesure, bénéficie de la grande stabilité d'une demande intérieure en croissance tenant à l'ampleur de ses besoins nationaux et à leur pérennité. En contrepartie, l'Inde se trouve confrontée à la nécessité de développer une industrie nationale pour répondre à des objectifs ambitieux que l'Agence ne peut plus satisfaire seule comme cétait le cas jusqu'ici. La transmission à l'industrie de la fabrication du lanceur PSLV mais aussi de satellites devenue inévitable présente toutefois le risque d'entrầner une augmentation des coûts et la nécessité de prendre en compte les logiques propres à dautres acteurs. Ce changement de culture va de pair avec l'annonce d'une possible ouverture à des investisseurs et des acteurs privés voulue par le Premier ministre Narenda Modi depuis son élection mais visiblement difficile à mettre en œuvre.

En 2019, la Chine et l'Inde sont désormais des puissances spatiales reconnues, l'effort chinois étant beaucoup plus marqué en termes d'investissement et témoignant d'une ambition de rattrapage plus rapide que celle affichée par l'Inde. Il s'agit maintenant d'analyser la façon dont chacune se positionne face l'émergence d'une nouvelle pratique des activités spatiales liée à lécosystème américain, avec l'intrusion d'acteurs privés imaginatifs dont les services de communication célèbrent les vertus d’entrepreneur comme l'incarnation de la quintessence de la philosophie américaine.

\section{Les ambitions chinoises et indiennes en 2019}

\subsection{Le contexte du New Space}

Si la dimension technologique symbolique des compétences spatiales perdure, de tout autres logiques sont désormais au centre de l'attention générale. La miniaturisation des composants spatiaux a été le premier changement majeur avec la mise au point des cubesats, utilisant un modèle standard de plateforme de la taille d'une boite de chaussures.24 Ces nouveaux satellites relativement

24 Un cubesat répond à des standards très précis: il mesure $10 \times 10 \times 10 \mathrm{~cm}$, pèse moins de $1,33 \mathrm{~kg}$ et a une puissance d' 1 W. Il est construit selon des spécifications ouvertes avec des composants en libre accès. Plusieurs cubesats peuvent être assemblés selon les besoins de l'utilisateur. 
faciles à réaliser ont popularisé l'usage de technologies spatiales à moindre frais et donc multiplié le nombre d'acteurs. La philosophie sous-jacente est celle de l'intégration de composants déjà testés pour des usages terrestres en renonçant aux très hauts critères de fiabilité initialement prescrits pour répondre aux contraintes physiques particulières du milieu spatial. Les résultats sont impressionnants puisque le nombre de satellites lancés double entre 2014 et 2017, dont $3 / 4$ ont une masse de moins de $250 \mathrm{~kg}$, les satellites de moins de $10 \mathrm{~kg}$ représentant à eux seuls la moitié de l'effectif.

Le deuxième grand changement a été la maitrise croissante des Big Data qui, alliée à la possibilité de mobiliser du capital risque, a permis à des entreprises indépendantes de proposer des approches répondant à la croissance attendue des besoins de transmission des informations et de développement des objets connectés. Différents projets de constellations d'observation de la Terre et de télécommunications sont ainsi en cours de réalisation modifiant à leur tour les formes à venir d'occupation de l'espace puisqu'il s'agit de milliers de satellites appelés à orbiter autour de la Terre. Le modèle américain de banalisation de lactivité spatiale poursuivi depuis le président Reagan et le Commercialisation Space Launch Act de 1984 semble désormais en passe de se réaliser au moins dans l'écosystème américain.

L'expression Alt.space, également utilisée pour insister sur la notion d'AlternateSpace" rend bien compte de cette approche se voulant révolutionnaire. Connue aussi comme Space 2.o ou New Space, ce nouveau paradigme affirme une redistribution des rôles entre acteurs privés et publics et une redéfinition des usages en lien direct avec les utilisateurs. Ce phénomène est cependant très inégal puisque ces petits satellites sont majoritairement américains, les satellites chinois étant 10 fois moins nombreux et les satellites indiens 50 fois.

Dans le domaine des satellites, l'irruption de multiples projets de constellations pour l'observation de la terre et les télécommunications est l'élément majeur pouvant modifier les équilibre en cours. Même si le modèle économique de ces nouvelles constellations nest pas encore testé, et qu'il faut envisager une baisse drastique par rapport aux réalisations, cela reste un indice de la maturité d'un secteur qui s'ouvre à des compagnies privées dont la culture n'est plus exclusivement aérospatiale mais intègre aussi celle des technologies de l'information. Lampleur de ce phénomène est toutefois difficile à analyser aujourd'hui. Il pose en tout cas la question de l'adaptation de la Chine et de l'Inde par rapport à ce nouveau contexte issu de l'écosystème US. 


\subsection{La position chinoise}

La Chine est souvent présentée comme le nouveau compétiteur des ÉtatsUnis. Le nombre de lancements annuels est souvent mis en avant pour témoigner de cette concurrence potentielle, Chine et États-Unis se disputant la première place depuis 2016. En réalité, ce critère purement numérique témoigne surtout de la rivalité latente dans tous les domaines qui caractérise actuellement la relation sino-américaine. Les capacités spatiales chinoises sont en effet bien loin d’atteindre les capacités américaines, le nombre de satellites ne préjugeant ni de leur durée de vie, ni de leur niveau technologique.

Cette comparaison est aussi utilisée comme ressort pour renforcer le volontarisme politique américain face à un concurrent d’autant plus redouté que les systèmes spatiaux nationaux sont explicitement présentés comme les garants de la sécurité nationale et une des clefs du leadership du modèle américain. Elle présente toutefois un autre aspect que l'on peut qualifier de boomerang en montrant à la Chine à quel point ses compétences spatiales sont redoutées et donc l'inciter à accroître son effort dans ce domaine.

Si l'on analyse la réalité des activités spatiales chinoises, le hiatus avec le secteur spatial américain est pourtant flagrant. Ainsi, la part de budget consacré à l'espace, estimée entre 5 et 7 milliards d'euros par an, contre 40 milliards pour les Etats-Unis confirme l'inégalité des moyens, d’autant plus que cet écart de dépense publique est récurrent depuis 60 ans. Nourries par une manne civile et militaire, les industriels américains disposent ainsi d'un potentiel de recherche et développement sans équivalent.

La question cruciale est celle des ambitions. Le Président Xi Jinping désigne explicitement le spatial comme un élément du rêve chinois qu'il promeut. Les réalisations dans le domaine des vols habités et les missions lunaires comme la mission Chang'E4 d'alunissage d'un rover sur la face cachée de la Lune fin 2018 s'inscrivent dans cette démarche. Elles servent une volonté de démonstration de compétences nationales aussi bien sur le plan intérieur qu'extérieur en proposant des missions originales impliquant une infrastructure technique inédite comme la mise à poste d'un satellite au point de Lagrange $e^{25}$ pour assurer les liaisons entre la Terre et la face cachée de la Lune. Mais les technologies utilisées sont encore loin des réalisations américaines. Alors que la Chine s’exerce à la robotique spatiale sur la Lune, les États-Unis font voyager la sonde américaine New Horizons aux confins du système solaire.

25 Les points de Lagrange sont des positions de l'espace où un objet reste stable par rapport au système formé par deux corps. 
De même dans le domaine des applications, le rattrapage par rapport aux pays occidentaux est en bonne voie et ce dans tous les domaines. Sont ainsi concernées au premier chef les télécommunications ou l’observation de la Terre, essentielles pour favoriser le développement économique global et diminuer les inégalités flagrantes dans la mise en valeur du territoire chinois perçues par le pouvoir politique comme un risque majeur pour la stabilité du pays. Plus récemment, les satellites sont mobilisés comme des supports au projet de Nouvelles routes de la soie (One Belt One Road, OBOR). Et les technologies spatiales sont aussi sollicitées dans le cadre du système de crédit social actuellement mis en place par le régime s'appuyant sur les technologies digitales appliquées aux comportements des citoyens.

Il est plus difficile d’apprécier la contribution précise des systèmes spatiaux militaires. Développés sous la responsabilité directe de la Direction générale des équipements militaires, ils sont encore relativement peu nombreux comparés à la flotte américaine. Les efforts de montée en gamme des matériels de l'Armée populaire de libération se traduisent néanmoins par un usage croissant des satellites, toutefois loin encore des performances américaines.

$\mathrm{Au}$ niveau international, la Chine est particulièrement active dans les forum internationaux. C'est ainsi que le 28 mai 2018, l'Office des Nations Unies en charge de l'espace (UNOOSA) invite en accord avec l'Agence chinoise des vols habités (CMSA) les États membres des Nations-Unies à proposer des expérimentations à bord de la future station spatiale chinoise (CSS) ${ }^{26}$. Cet appel, dont il n'existe pas déquivalent, démontre la volonté chinoise de reconnaissance en tant que nouvel acteur majeur et son usage de la tribune des Nations Unies pour le faire savoir.

Il est intéressant de noter d'emblée la parfaite maîtrise par la Chine de l'actualité spatiale. En effet, la station chinoise est supposée opérationnelle à partir de 2022 tandis que les États-Unis annoncent la fin de l'ISS, la station spatiale internationale, pour 2025. Les participants actuels et les éventuels futurs candidats sont donc confrontés depuis plusieurs mois à la délicate question de savoir comment maintenir leur compétence dans le domaine des vols habités. La Chine apparaît alors à peu de frais comme un partenaire incontournable pour des agences nationales naturellement désireuses de profiter d'une telle occasion. En parallèle, elle récupère le rôle de leader précédemment tenu par les

26 Disponible à: <http://www.unoosa.org/oosa/en/informationfor/media/2018-unis-os-496.html>. Accès en : 03 juin 2019. 
États-Unis et la Russie qui a assuré la permanence des vols habités et dont les modules issus de la station Mir ont été la base de la station.

De plus, cette annonce est l'occasion pour l'UNOOSA de célébrer la puissance spatiale de la Chine et, pour l'ambassadeur chinois aux Nations Unies, Shi Zhongjun, d'affirmer l'attachement de la Chine au Traité de $1967^{27}$ et à la libre utilisation et circulation dans l'espace au profit de toute l'humanité. Une telle position dépasse évidemment le seul domaine de loccupation humaine de l'espace et renvoie à la volonté chinoise, en coopération étroite avec la Russie, de lutter contre la militarisation de l'espace ${ }^{28}$ et la politique américaine de contrôle de l'espace.

La politique spatiale chinoise repose donc sur une double approche: répondre aux besoins nationaux et conforter son image internationale. Dans cette perspective, la Chine ne peut se tenir à lécart des nouvelles formes d'occupation et en particulier de la place tenue par les acteurs privés. On voit ainsi apparaître quelques nouveaux acteurs dont la logique s'inspire de celle des entrepreneurs américains mais qui restent proches de groupes étatiques, régionaux ou intégrés dans des réseaux sous contrôle du pouvoir. C'est sans doute par le biais des efforts du gouvernement chinois pour promouvoir l'innovation que des acteurs/ entrepreneurs du spatial pourraient progressivement jouer un rôle complémentaire de celui tenu par les instances publiques.

\subsection{La position indienne}

L'intégration de la communauté spatiale indienne dans les réseaux internationaux, la structure du secteur spatial et la nature civile des programmes spatiaux distinguent nettement l'Inde de la Chine.

C'est ainsi que l'Inde a défrayé la chronique spatiale avec un nombre record de petits satellites (104) lancés en un seul tir du PSLV en février 2017 apparaissant ainsi comme un acteur important du New Space. Pourtant l'intérêt manifesté au niveau national pour le segment des petits satellites en tant que tel reste faible. Plus largement les entreprises du New Space indien, peinent à trouver leur place dans lécosystème national. En effet, l'originalité du cas indien - avec le rôle spécifique de l'ISRO comme agence en charge de la réalisation des programmes - se traduit par une extrême faiblesse de l'industrie nationale spatiale.

27 Le traité sur les principes régissant les activités des États en matière d'exploration et d'utilisation de l'espace extra-atmosphérique, y compris la Lune et les autres corps célestes, ou le traité de l'espace, est un traité ratifié en 1967 relatif à l'exploration et à l'utilisation de l'espace extra-atmosphérique. 
La difficulté à trouver des investisseurs nationaux et la complexité pour accueillir des investissements directs étrangers dans un contexte politique qui reste confus ne permettent pas de dépasser ces limites.

Les activités spatiales indiennes en 2018 sont marquées par deux slogans particuliers du gouvernement Modi: Make in India et Digital India. Le second a une implication immédiate sur le développement d'infrastructures spatiales puisqu'il s'agit de connecter l'ensemble des zones rurales qui représentent plus de la moitié des 500 millions d'utilisateurs prévus dans le cadre des programmes tels que la e-gouvernance et la reconnaissance par données biométriques des citoyens. ${ }^{29}$

Compte-tenu de ces besoins et de la géographie de l'Inde avec une population dispersée dans des régions isolées d'accès souvent difficiles, le satellite présente des atouts évidents. Les experts indiens du spatial mettent ainsi en avant leur expérience dans le domaine des télécommunications et la disponibilité enfin acquise du lanceur GSLV qui doit permettre un coût encore réduit dans la mise sur orbite de satellites géostationnaires. Ils soulignent par ailleurs la possibilité d'utiliser des constellations en orbite basse sur le modèle des projets américains. La difficulté qui demeure, et elle n’est pas des moindres, est la capacité du secteur spatial, en l'occurrence l'ISRO, à développer ces satellites et à maîtriser les technologiques avancées nécessaires à leur réalisation.

Parallèlement aux tentatives de l'ISRO pour résoudre le goulet détranglement des télécommunications, l'observation de la Terre représente le point fort du spatial indien. Les responsables de l'ISRO soulignent ainsi volontiers que la constellation indienne des satellites IRS est la plus importante des flottes gouvernementales, ce qui renforce encore d'ailleurs la singularité du cas national.

Le défi principal auquel est aujourd'hui confronté le spatial indien est de répondre à une demande qui ne cesse de croitre. En 2017, plus de 170 projets portés par 60 entités gouvernementales concernaient aussi bien la gestion des ressources naturelles que l'énergie et les infrastructures, la prévention des risques majeurs, la navigation, les flux de télécommunications, la e-gouvernance... bref la gamme complète des applications spatiales civiles mais aussi duales, et désormais aussi liées à des enjeux de sécurité.

Dans les faits, de telles ambitions se traduisent par la programmation de 71 satellites à construire d'ici 2021 et à lancer avec une fréquence atteignant les 18 tirs par an.

29 Le Aadhar number permet à chacun de disposer d'une identité reconnue par l'ensemble des systèmes publics. 
Même si des raisons budgétaires compte tenu de la modestie du budget indien ( 1,8 milliard de dollars en 2018) peuvent entraîner une baisse de ces prévisions, la tendance demeure et interdit de poursuivre sur le modèle actuel d'une agence dont une part croissante des ressources en hommes et en infrastructure serait consacrée à des activités strictes de production.

Différentes logiques sont désormais à prendre en compte. La première est celle du transfert de compétences à l'industrie qui devient dès lors un acteur à part entière. Ce basculement pose la question du partenariat mais aussi de la rentabilité et donc en creux, à terme, celui de la commercialisation du fait de l'ISRO (plus précisément d'Antrix) ou des acteurs industriels.

A ce titre, le secteur spatial indien se retrouve à un tournant de son histoire. La situation actuelle est l'héritage de la volonté d'autonomie d'une bureaucratie spatiale dont les succès sont reconnus et dont la validité des choix dans la définition de ses objectifs n'est pas mise en cause. Les réalisations spatiales indiennes se caractérisent par une vision de type service public qui a fortement contribué à la légitimité du secteur. Le secteur spatial a calculé ses bénéfices essentiellement au travers de retombées indirectes, sectorielles ou globales: efficacité plus grande de la pêche, actions d’aménagement sous différentes formes comme la recherche d'eau, de matières premières, le suivi des récoltes, la télé-éducation ou le télé-enseignement, une commercialisation directe de produits spatiaux peut aussi être bienvenue pour renforcer sa position. Par ailleurs, la diversification des activités (exploration, vols habités) que l'ISRO ambitionne désormais ne sera pas sans conséquence. Les acteurs du spatial sont bien conscients du fait qu'ils ne pourront pas obtenir de hausses exagérées de leurs ressources. L'intervention d'acteurs extérieurs représente un facteur de perturbation que les responsables ont voulu éviter le plus longtemps possible. Il leur faut cependant désormais envisager de concentrer leurs compétences là où elles sont les plus nécessaires et accorder une dévolution plus grande à l'industrie.

La dimension internationale est l'autre grande donnée originale du spatial indien historiquement construit sur la coopération. Pour autant, le monde actuel n'est plus celui de la guerre froide et la position non alignée de l'Inde a été amenée à évoluer.

Dans le cas du spatial, l'Inde reste soumise aux règlementations en matière de transferts de technologies et elle est encore loin de l'indépendance de la Chine en la matière. Il lui faut donc tout à la fois préserver de bonnes relations avec les fournisseurs de technologies modernes pour ses satellites d'application, en particulier les États-Unis et l'Europe. Pour autant, l'Inde n'est pas prête à prendre le risque de renoncer à son partenariat ancien avec la Russie d'autant 
plus qu'elle reste intéressée par les compétences de cette dernière dans le domaine des missiles en particulier.

Par ailleurs, l'Inde est désormais une puissance spatiale suffisamment accomplie pour vouloir pratiquer aussi sa propre diplomatie spatiale même si celle-ci reste encore embryonnaire. Cette approche se décline sur différentes aires; régionale afin de contrebalancer autant que faire se peut l'influence de la Chine, Sud-Sud conformément à sa volonté de promouvoir le multilatéralisme et internationale dans le cadre de l'affichage progressif de son soft power.

La relation avec la Chine est au cœur des préoccupations indiennes dans tous les domaines. L'importance des échanges commerciaux entraîne des efforts de normalisation réels avec l'organisation de visites officielles croisées, mais lévolution de la puissance chinoise et son rôle global croissant représente pour l'Inde une menace scrutée en permanence. Largument de la concurrence potentielle avec les avancées chinoises dans le domaine de la science et technologie est ainsi systématiquement repris dans les réflexions politiques sur le devenir du spatial indien.

Cela favorise la relation avec le Japon qui recherche aussi de nouveaux partenariats et la mise en avant de synergies pour des applications spatiales comme la surveillance maritime et plus largement les technologies duales qui pourraient contribuer à accroître la sécurité régionale.

L'Asia-Pacific Regional Space Agency Forum (APRSAF), une initiative japonaise de la JAXA et du ministère de l'Éducation, de la Culture, des Sports, des Sciences et de la Technologie du Japon (MEXT), trouve en effet un écho croissant du côté de Delhi. Cest ainsi que le meeting de 2017 sest tenu en Inde avec la contribution de l'ISRO. Les thèmes privilégiés répondent aux préoccupations mises en avant par l'Inde dans ses propres programmes, en particulier, la question du climat et des risques naturels, de même que la formation aux utilisations spatiales et aux services aval.

Le spatial est ainsi un élément du soft power indien. Les lancements par le PSLV de micro et petits satellites sont volontiers mis en avant au niveau national pour montrer la maturité de la puissance spatiale indienne, même si les revenus générés par ce type de lancements sont plus symboliques que financiers. Cela permet en tout cas à l'Inde détablir des liens avec les communautés surtout scientifiques de pays du Nord et du Sud qui correspondent parfaitement à son affichage de multilatéralisme.

Enfin, fidèle à son attachement aux enceintes internationales, l'Inde a répondu aux appels à propositions du sous-comité scientifique et technique du COPUOS de février 2017 dans le cadre d'UNISPACE. Le programme de formation 
au développement de petits satellites au travers de cours théoriques et d'expérimentations proposé par l'ISRO via l'U.R. Rao Satellite Centre a été retenu et intégré dans la conférence UNISPACE+50 organisée à l'occasion du cinquantenaire de cette initiative sur le thème du développement durable. Pour l'Inde qui avait entamé son programme spatial dans cette enceinte, la coopération affichée de l'ISRO et de l'UNOOSA revêt une valeur particulièrement symbolique.

\section{Conclusion}

Conformément à leur image de puissance du XXIe siècle, la Chine et l'Inde sont appelées à jouer un rôle de premier plan dans le monde spatial. Cependant, leur organisation tant politique qu'industrielle, comme leur place particulière sur la scène internationale ont pour conséquence des priorités nationales différentes. C'est finalement dans la recherche d'une influence régionale significative que la Chine et l'Inde manifeste le plus clairement leurs différences mais aussi leurs ambitions.

\section{Références}

ABDUL KALAM, Avul Pakir Jainulabdeen. Wings of Fire, Hyderabad, Universities Press, 1999.

CHEN, Kuan-Wei; JAKHU, Ram S.; NYAMPONG, Yaw, Global Space Governance, Montréal, McGill University CRASL, 2015.

CHAO, Chien-min; DICKSON, Bruce J. Remaking the Chinese State: Strategies, Society and Security, New York, Routledge, 2001.

COULOMB, Jean. L’Année géophysique internationale (1957-1958). L’Astronomie, v. 70. Disponível em: <http://articles.adsabs.harvard.edu/cgi-bin/nph-iarticle_query?19 56LAstr..70....1C\&amp;data_type=PDF_HIGH\&amp;whole_paper=YES\&amp;typ $\mathrm{e}=$ PRINTER\&amp;filetype $=. p d f>$. Acesso em: 07 maio 2019.

HARVEY, Brian. China in Space: the Great Leap Forward, London, Springer/Praxis, 2013. JAKHU Ram S.; JASANI, Bhupendra. Commercialisation of Space: Opportunities and Challenges. Bangalore/New Delhi, NIAS \& Pentagon Press, 2015.

MCDOUGALL, Walter A.... the Heavens and the Earth: A Political History of the Space Age. Baltimore, Johns Hopkins University Press, 1997.

MANORANJAN RAO, PV; SURESH, BN; BALAGANGADHARAN, VP. From Fishing Hamlet to Red Planet: India's Space Journey. ISRO, e-book, 2015.

RAJAGOPALAN, Rajeswari; JOHN, Arvind. A New Frontier: Boosting India's Military Presence in Outer Space. New Delhi, Observer Research Foundation, 2014.

RAO, Udupi Ramachandra. India's Rise as a Space Power. New Delhi, Cambridge University/Press India, 2014. 
SOURBÈS-VERGER, Isabelle; BOREL, Denis. Un Empire très céleste, la Chine à la conquête de l'espace. Paris, Dunod, 2008.

SOURBÈS-VERGER, Isabelle (dir.), "L'espace en jeu”, L'Information géographique. Paris, Armand Colin, Juin 2010, v. 74, n. 2.

VERGER, Fernand (Org.). The Cambridge Encyclopedia of Space. Cambridge, Cambridge University Press, 2003.

Recebido em 22/02/2019

Aprovado em 18/06/2019

\section{Como citar este artigo:}

VERGER, Isabelle Sourbès. Nouvelles formes d'occupation de l'espace, la place de la Chine et de l'Inde. Contemporânea - Revista de Sociologia da UFSCar, v. 9, n. 1, jan.jun. 2019, pp. 79-98. 